\title{
A Systematic Review of Influenza Forecasting Studies
}

\author{
Jean-Paul Chretien*1, Dylan George ${ }^{2}$ and Ellis McKenzie ${ }^{3}$ \\ ${ }^{1}$ Armed Forces Health Surveillance Center, Silver Spring, MD, USA; ${ }^{2 B}$ Biomedical Advanced Research and Development Authority, \\ Washington, DC, USA; ${ }^{3}$ Fogarty International Center, Bethesda, MD, USA
}

\section{Objective}

To assess studies of epidemiological forecasting models for human influenza activity.

\section{Introduction}

Researchers have developed varied methods for forecasting influenza activity using surveillance data with predictive models, but real-world applications in public health programs are rare. To inform consideration of whether and how public health practice should incorporate influenza forecasting, we conducted a systematic review of these methods.

\section{Methods}

We used the PRISMA methodology [1] to identify papers that: described dynamic forecasting models [2] (i.e., use surveillance data collected during an epidemic to forecast the course of the epidemic) for influenza or influenza-like illness (ILI) activity in human populations; and validated models against independent data (real or simulated). We searched 3 databases (PubMed, CINAHL, Project Euclid) for publications in English since 2000 using the query: influenza AND (forecast* OR predict*) (any search field). We screened all titles and abstracts, and reviewed the full text when needed to identify papers meeting inclusion criteria. For selected papers, we recorded application type (seasonal or pandemic influenza), epidemiological setting (population-based, hospital-based, or regional/global pandemic spread), geographic setting, forecast type (temporal or spatio-temporal), forecast method, surveillance input data, validation results, and type of sensitivity analysis.

\section{Results}

The database search identified 2,359 papers. After screening titles and abstracts, we selected 137 for full-text review, and included 36 of these, summarized in the Table.

Among population-based and hospital-based studies $(\mathrm{N}=28$ and 4 , respectively), 10 used ILI data only, 8 used virological data only, and 2 used both ( 1 of these also used serological data). Four studies used internet search query data in addition to or instead of clinical surveillance data (Google Flu Trends, N=3; Baidu, N=1). Five studies used meteorological data in addition to influenza-related data. The regional/global pandemic spread studies $(\mathrm{N}=4)$ used data on the time and location of pandemic (H1N1) 2009 emergence to forecast spread to other countries.

While many studies compared models with different predictors, only 1 compared a (more complex) individual-based model to a (simpler) compartmental model. Eight studies presented results of sensitivity analyses.

Eight population-based studies measured the accuracy of peak activity week forecasts made 4 or more weeks before the actual peak, with median prediction error ranging from -4.5 to 0 weeks. In 3 hospital-based studies, models provided 1 -step-ahead forecasts of influenza or ILI incidence (step $\leq 1$ month). In 2, prediction error was $\leq 20 \%$; in the other, $83 \%$ of predictions were within $20 \%$ of observed values. Of the regional/global pandemic spread studies, 1 predicted pandemic 2009 (H1N1) peak activity and 1 predicted arrival time across countries globally, with peak week prediction error $\leq 4$ weeks for $95 \%$ of countries and correlation between predicted and observed arrival time $=0.62$, respectively.

\section{Conclusions}

Studies have applied influenza forecasting to diverse geographic and epidemiological settings; many reported results suggesting real-world usefulness. However, lack of sensitivity analyses and direct comparisons of different model types, and implementation challenges for individual-based and more complex compartmental models, may hinder translation from research to public health practice.

Overview of influenza forecasting studies

\begin{tabular}{|c|c|}
\hline Study characteristic & No. studies (N=36) \\
\hline Application & 22 \\
\hline Seasonal influenza & 12 \\
Pandemic influenza & 2 \\
Unspecified & 28 \\
Epidemiological setting & 4 \\
\hline Population-based & 4 \\
Hospital-based & 15 \\
Regional/global pandemic spread & 14 \\
Geographic setting[a] & 5 \\
\hline Europe & 3 \\
\hline North America & 29 \\
Asia/Pacific & 7 \\
Global & 19 \\
\hline Forecast type & 12 \\
\hline Temporal & 5 \\
\hline Spatio-temporal & Forecasting approach \\
\hline Curve prediction: time series, GLM, Bayesian network, other & \\
\hline Diffusion model: compartmental & \\
\hline Diffusion model: individual-based &
\end{tabular}

[a]Some studies included $>1$ location.

\section{Keywords}

influenza; forecasting; prediction

\section{Acknowledgments}

The views are the authors', not necessarily those of the US Departments of Defense or Health and Human Services.

\section{References}

1. Moher D, et al. Preferred reporting items for systematic reviews and meta-analyses: The PRISMA Statement. PLoS Med 2009;6:e1000097.

2. Hyder A, et al. Predictive validation of an influenza spread model. PLoS ONE 2013;8:e65459.

\section{*Jean-Paul Chretien}

E-mail: JPChretien@gmail.com 